This is the accepted version of the paper. This version may differ from the final version published by SpringerLink. The final authenticated version is available online at DOI: https://doi.org/10.1007/978-3-030-49282-3_4

\title{
Co-creating persona scenarios with diverse users enriching inclusive design
}

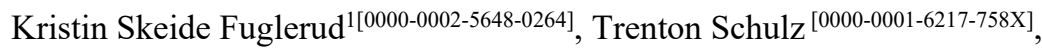 \\ Astri Letnes Janson ${ }^{20000-0002-7730-7372]}$ and Anne Moen ${ }^{3[0000-0002-3825-9355]}$ \\ ${ }^{1}$ Norsk Regnesentral, Postboks 114 Blindern, 0314 Oslo, Norway \\ ${ }^{2}$ Division of Diagnostics and Technology, Akershus University Hospital, Norway \\ ${ }^{2}$ Institute of Health and Society, University of Oslo, Norway
}

\begin{abstract}
In this article, we will examine personas as methodological approach and review some critiques about how its use may omit or stereotype users with disabilities or even restrict user involvement. We review previous persona creation methods and compare it to our approach where we involve diverse users directly in the personas creation process, to ensure more grounded personas. This approach has recently been refined in a project where we are building a tool aiming to give citizens more control over their health information. We discuss our experiences and offer some experience-based guidelines for using our method.
\end{abstract}

Keywords: Co-design, Participatory design, Universal design, Inclusive design, People with disabilities, Health information management, Patient engagement

\section{Introduction}

The concept of personas has been around for over 20 years [1]. Personas are "fictitious, specific, concrete representations of target users" and are used to keep people in mind throughout the design cycle. Success stories are reported [1,2], but each team has documented different ways of using personas. Common claims are that personas help visualize a typical user, support empathy, and bring designers closer to understand the users they will be designing for. The premise is that understanding at least one typical user should result in a better product or service than designing for a general user whose abilities may change depending on the preferences of the designer.

Experts that have experience with using personas assert that some of the most important benefits are that (1) personas supports audience focus through challenging assumptions, prevention of self-referential design, and (2) that personas help in prioritizing product requirements and product audience [3]. It is recommended that personas are generated from aggregated user research, combining input from many users into a narrative form [4]. Personas can be built on both qualitative and quantitative data, such as interviews, focus groups, questionnaires and demographics. Because humans are naturally good at modelling other people, personas can be more engaging 
and memorable than many other representations of user research [5]. When personas are based on data from activities involving users it can be a way of giving users a "voice" or a say in a project similar to user-centered or participatory design [6]. Users, however, do not normally participate in the persona creation process itself.

If personas are used to stand in for the typical user, how will they function for the atypical user? That is, how well can personas stand in for or "replace" persons with disabilities, especially if the persona creation process normally excludes the users? How can we set up co-creation processes that ensure participation of people with disabilities and foster a deeper understanding by attending to and including the needs of persons with different disabilities?

In this article, we will examine the use of personas for design purposes (Section 2) and focus on a method for co-creating personas that also include disabilities (Section 3 ). Then, we look at some common critiques to the personas approach (Section 4). This will be used to compare to the method we recently used in a project where we are building a tool for giving citizens more control over their health information (Section 5). We end with a discussion of this method and guidelines for others to implement the method themselves (Section 6 and Section 7).

\section{Persona use in research and the industry}

Personas have been used in many research projects and lead to successful product developments. One of the goals of introducing the personas concept was to make designers focus design efforts on people who were not similar to themselves. Cooper [1] documented his idea of personas and how the products created via his technique have been used by many people.

Grudin and Pruitt documented three years of the use of personas inside Microsoft for a variety of projects [2]. Their colleagues and themselves had built on the personas process and asserted that personas helped engage team members, helped uncover design issues that other methods could not find, and could enhance the scenarios in a project by becoming the actors inside them. Earlier, Pruitt and Grudin [7] also argued that personas could be a powerful tool for participatory design, since creating personas required designers to engage with the people that would use the product and the work those people do. They warned, however, about reusing personas, since personas are created for a specific purpose with specific and situated choices and reusing the personas may stretch them and blind designers to contradictory information about the new design situation. This has led to textbooks that provide a detailed description of how to create and use personas $[4,8,9]$.

Personas are increasingly used in the design of digital health services as well [10, 11]. Personas and scenarios are proposed as communication tools for multidisciplinary teams developing medical devices [12]. There is limited literature on co-creating personas with users. Donald et al. [13] had positive experiences of using personas in a consensus workshop with patients, and suggests using personas in patient-oriented research to explore their needs and preferences. Valaitis et al. [14] have involved patients and clinicians in persona-scenario workshops for a primary care intervention. 
They conclude that this presents a promising alternative to active engagement of multiple stakeholders in the co-design of complex health interventions.

Although personas are normally used for product design and human-computer interaction (HCI), personas have found their way into fields like human-robot interaction (HRI). Specifically, personas have been created and formalized as models for the robot to know how to interact with a person [15].

Methods for creating personas have also been applied to create personas for people with disabilities. Some of the authors have documented a process used in several projects that created personas with different disabilities and different types of Assistive Technology [16].

The ÆGGS project (http://www.aegis-project.eu/) created a number of personas with different types of disabilities $[17,18]$. These personas are available to others for inspiration to use in their projects. While some researchers do not recommend reusing personas, others may think that providing research-based personas to designers that do not have access to users with disabilities can be helpful. Others have created a set of personas that match a wide range of European Older Adults skills and disabilities based on demographic data [11, 19]. Gonzalez de Heredia et al. [11] propose quantitative measures to design personas for healthcare development and policy-making, based on several surveys of the aging population.

There have been some novel uses personas with disabilities. One project has used personas with disabilities as a guide for creating accessible content in Massively Open Online Courses (MOOCs) [20]. Participants would receive a persona description and attempt to create content that would be accessible to the persona's disabilities. This work was then checked by a peer who had access to the same persona. The HRI persona model has expanded to use a method for creating personas with disabilities [16] to create models of personas with dementia and paralysis [21]. Let us examine that method.

\section{Previous method for creating personas with disabilities}

Schulz and Fuglerud have presented their method for method for co-creating personas with disabilities [16]. This process consists of gathering information from people with disabilities, looking at the assistive technology (AT) that is used by people with disabilities, running a persona workshop with stakeholders, creating a persona skeleton based on the results from the workshop, and finally writing up the actual personas.

To gather information, Schulz and Fuglerud recommend combining standard usercentered design methods like observation, focus groups, interviews, and surveys. They note that when organizing for interview or focus group, it is important to carefully pick well-known locations that are easy to reach for people with disabilities. Similarly, an online survey tool needs to be universally designed so that it works with the AT people use to answer the questions. Knowledge about AT adds important insights, and Schulz and Fuglerud stressed the value of someone in the design team had actual experience working with people with disabilities, for example by learning to use an AT or by participating in user tests involving people using AT. If the team did 
not have a person with such knowledge, one could invite such a person as part of a persona workshop.

The persona workshop gathered stakeholders to help generate the beginning of the personas. The method described by Schulz and Fuglerud builds personas based on assumptions and factoids and is based on work from Adlin and Pruitt [4]. Assumptions are quotes, opinions, or goals that could come from a potential persona. These assumptions are usually based on the knowledge or experience of the stakeholder. Factoids are small facts or news articles that come from background research and material that is available at the workshop. Participants begin by looking through and rereading the background material, writing down factoids on items that are easy to rearrange (for example, writing one factoid on a post-it note, or nodes in a mindmapping software). When the rate of extracting factoids slows enough, participants then begin writing assumptions. For each assumption, participants also add a possible persona name who may have this assumption.
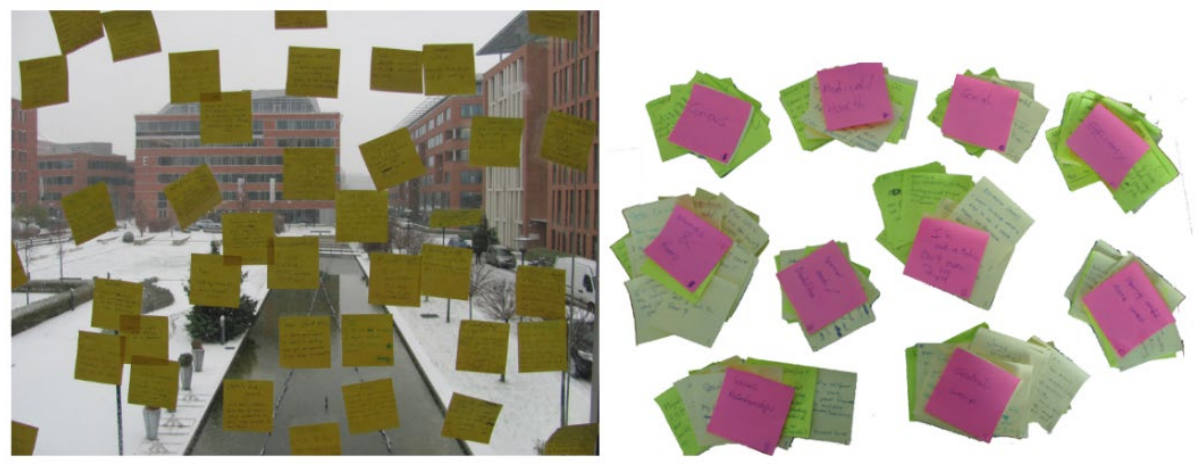

Fig. 1. Examples of placing assumptions and initial clustering (left); these assumptions are later grouped with factoids into groups and themes are identified for persona skeletons (right).

When all the participants have finished with the factoids and the assumptions, the participants begin to build groups of similar assumptions (Fig. 1., left). Once all the assumptions have been put into groups, these groups are used as the starting point for grouping factoids. This usually results in another regrouping.

When this regrouping is finished, the groups are identified as themes (Fig. 1., right) and are used as a starting point for persona skeletons. Persona skeletons begin as an outline that contain the themes and keywords based on the assumptions and factoids from the different groups. This makes it easier to communicate between stakeholders to understand what the personas are going to look like and suggest changes. Once the basic skeletons are decided, they are filled out with more information. For example, a story may be developed for a persona to flesh out why that persona has a particular assumption with factoids providing a basis for the story. During this process, the personas presentation begins to take shape, and the persona's AT must also be selected and described, including the persona's expertise in using the AT.

Although Schulz and Fuglerud recommend co-creating personas with stakeholders, they do not explicitly include people with disabilities in that stakeholder group. 


\section{$4 \quad$ Persona critiques}

One frequent critique of personas is how they are used in the design effort. Cooper originally claimed that some designers would take a personas side and argue that a persona would never perform a particular action [9]. Bødker and her colleagues, however, found that personas didn't necessarily result in designers (or anyone) taking the persona's side, and that they may draw attention away from real participation of actual users [22]. Others have found that even projects that do not create personas end up with some designers creating an ersatz persona in their heads, with limited relevance for the users they sought to support [23]. One study found that practitioners used personas almost exclusively for communication, and not for design [24].

Some authors claim that personas with a personality reduces the need for human participants during design [25]. Others argue that personas cannot completely replace immersion in actual user data [24]. Using personas with some disabilities can even give the project group a false sense of understanding users, whereas, in reality, it creates an extra layer of interpretation between users and developers and thus can create a greater distance [26].

Another critique of personas is how they are created. Even though there are articles and textbooks on the subject [4], designers create personas in different ways [23]. Cooper himself laments that the one chapter he wrote to introduce the persona technique [9] was incomplete [1]. Given the different methods of persona creation, this raises the question of how representative and reliable persona is? That is, how much of a persona is real versus imaginary?

One way of ensuring representative personas is to base them on empirical and statistical data [7]. A statistically valid common behavior may stand stronger than an isolated account with unknown occurrence frequency [22]. Data-driven persona creation carries a risk that the researchers only look for data that confirms their assumptions. This results in the design of an ideal persona rather than a realistic one [27]. The data may also be contradictory, and some have found it difficult to remain true to a creation methodology when encountering contradictory evidence [28].

There is another reason to be cautious of only using quantitative data when creating a persona with disabilities: people with disabilities are rarely part of a statistical average, instead they are typically statistical outliers. Treviranus [29] has argued that creating personas based only on statistics and background research will not teach a designer enough about the individual struggle of a person with disabilities. An important concern in inclusive design is therefore to involve marginalized users in the design process [30]. Thus, from an inclusive and universal design perspective two main issues are how to generate data from users, and whether users themselves participate in their creation. Having users participate helps combat selection bias and imposing assumed traits, experiences and challenges over involving users directly.

The method described by Schulz and Fuglerud [16] set out to create personas with disabilities. However, it also can lead to processes where people with disabilities do not actively participate in the creation process. For example, feedback from people with disabilities might be restricted to before the persona workshop begins, rather than people with disabilities actually being part of the workshop. The people with 
disabilities feedback could be along as research material for the factoids, but the people themselves may be absent. Similarly, the AT expert can join the workshop, but this expert may only know how the AT works and not have to live with the AT as a way of interacting with the world. In addition, the most common method of creating assumptions and factoids (i.e., writing them on post-it notes and grouping them) may exclude people with reading, writing or mobility disabilities (e.g. people who are blind or have limited hand-function). It is not impossible to overcome these obstacles, but it requires planning and organization. Thus, we wanted to investigate whether involving users in the personas creation process could help counter some of the identified challenges, while retaining the main strengths of personas.

\section{$5 \quad$ Involving users with disabilities in creating personas}

In this section, we present our experiences with including users in the persona scenario creation process as part of the ongoing CAPABLE project [31]. The aim of the CAPABLE project is to create a digital tool that enable citizens to actively use their clinical and personal health information. The demonstration of the tool concentrates on three areas; medication, nutrition, and coordination of health service information.

An important goal for the CAPABLE solution is that it shall follow the principles of universal design, meaning that it shall be accessible and usable to as wide a range of people as possible, including people with disabilities. Therefore, the development of the CAPABLE tool is based on an inclusive user-centered design approach [30], emphasizing user involvement throughout the development cycle.

In the CAPABLE project, we follow a qualitative and interpretive research approach using different user-centered methods. In addition to five personas workshops, described in more detail below, we have run several other activities: (1) two focus groups, one with seven participants from an elderly council, and one with six participants from a disability council; (2) a design workshop with twenty-four young people, divided into five groups; (3) a pluralistic usability walkthrough of a low-fidelity prototype with four seniors from another senior council; and (4) a paper prototype user testing with a senior citizen. The participants represent citizens: (a) from adolescence to elderly, (b) with and without disabilities, (c) with varying degrees of health problems, (d) digital health literacy, and (e) in different stages of life: pupils, workers, next of kin and retirees. The participants were recruited through three municipalities that are partners in the project. The municipality recruited participants from their existing municipality councils (elderly, disability, youth).

For the persona workshops (Fig. 2), users were recruited from three nongovernmental disability and health advocacy organizations (NGOs), which are also partners in the project. The plan was to develop six persona scenarios through two workshops in each NGO. We wanted the personas to be diverse in terms of sex, age, type of health challenge, disability and demonstration area (medication, nutrition and coordination of health service information). These parameters were therefore distributed across six very rough persona skeletons. 
We ended up with five persona scenario workshops with different user groups recruited from the three NGO's: people with rheumatism, people with Chronic obstructive pulmonary disease (COPD), people with cardiovascular diseases (CVD), people with low vision, and people who are blind. There were three users in each workshop, fifteen in total. The workshop lasted around 2-3 hours, including a break with some food, and the participants got a gift card as a compensation for their contribution.

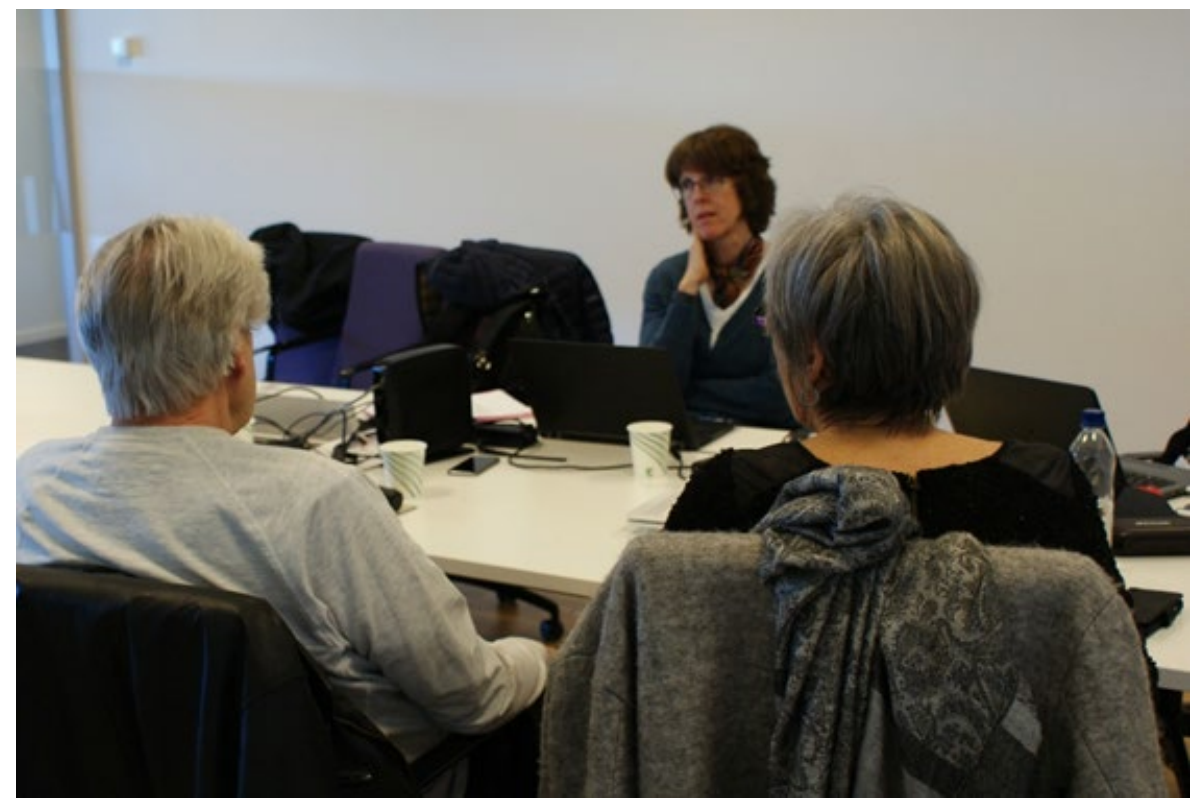

Fig. 2. Co-creating persona scenario with users

The workshops were divided into two parts. In Part One, the persona concept was presented to the users. Their task was to create a fictional person that could be their peer, with realistic characteristics and challenges. They were introduced to the rough persona skeleton as a starting point, and were encouraged to discuss and create a mental picture of their persona, including the physical appearance, social environment, education, work, personality, interests, values, and information about health challenges and disability.

In Part Two, the persona was extended further with a focus on their health history, with details about medication, coordination, and food information needs. We explicitly asked the participants to think of concrete episodes and stories that could highlight challenges with health information management in the three demonstration areas for CAPABLE. While we wanted the persona description to be realistic, we would allow for the persona to have experienced somewhat more challenging situations than the participants considered usual for one single person. The aim of this was to encourage the participants to think of and discuss various situations that could be of relevance. Finally they were challenged to create scenarios for how they envisage that the CAPABLE tool could help the persona with some of the identified challenges. 
The discussions were audiotaped and detailed notes and audio transcripts were used as a support for writing up the personas. For simplicity, we refer to the resulting persona descriptions including their health history and potential future CAPABLE usage scenarios as the persona scenario. These persona scenarios were distributed to the participants for comments and corrections. For documentation of user needs and to inform the design, we conducted a thematic analysis based on detailed field notes from all the activities, including the persona workshops. This is included in the basis material for the development of requirements for the CAPABLE tool.

We found that the discussions in the persona workshops provided detailed insight into the needs of the persona, and also of other persons with a similar disability or condition. Because the participants had experience from participating in peer-support and advocacy work through the NGOs, they could draw upon their insight into experiences and challenges among people with similar disabilities and conditions as themselves.

The discussions about the persona health history and various information needs and challenges were particularly detailed and provided useful insights and information for the project, and a different type of information than the information from the focus groups or usability walkthrough. There were quite some overlap between the themes emerging from the personas workshops and from the other methods. While the discussion in the focus groups often were quite high level, the usability walkthrough and design workshop gave concrete and detailed feedback to our PowerPoint prototype. The persona workshops on the other hand, gave detailed insight into concrete challenges during the life of the personas. The collected data represented a more coherent narrative, which showed challenges in dealing with health information over time and from the perspective of a person with a particular type of disability or health condition. This conversation was particularly different between focus groups and personas workshops.

The users' immediate feedback on the method was that it was fun and engaging. In the personas workshops, participants could talk about challenging experiences without disclosing one's or others' personal information. This made it easier to discuss potentially difficult and vulnerable issues. After all, the other participants could not know whether it was the participant's story or someone else's that they knew. In the focus groups, participants hesitated considerably to disclose their experiences. In both settings, the participants knew each other from before. Beyond the method, the number of participants present was the main aspect differing the two. This is another factor that could help explain the difference in the type of information that were discussed.

\section{Discussion}

As many have pointed out, there are inherent conflicts and tensions when trying to condense rich qualitative and quantitative data along many different axes into one or a few personas. Several authors have discussed the difficulty of creating a representative, coherent and believable personas based on such material, risking to create what 
Bødker describes as a kind of Frankenstein persona [22], or a persona that nobody is able to relate to. The idea of creating one or a few average personas to represent a large segment, will in a way break with the original idea of personas, namely that it is better to really understand and design for one person than to try to design for a general user. When reducing such materials into a few personas we are likely to lose important insights about people who are statistical outliers, such as people with disabilities, or people with special health conditions, and about time and context aspects that are important to understand their lived experience.

Some may dismiss having users participate in persona scenario creation as difficult or confusing for the users, or that it may be difficult to organize. Users in our workshops, however, quickly grasped the idea of personas scenario. Users seemed to enjoy themselves, participating actively in the workshop. One participant commented that she was reluctant in retelling her personal health history in a group, because it could be too personal and meant reliving traumatic events around her condition. She found it much easier and helpful for the co-design workshop to talk about such events in a fictive third person perspective, and create a persona scenario based on different stories she knows.

We experienced that co-creating personas scenarios, including a health history and future tool usage scenarios, provided a type of information that we could not derive from focus groups or usability walkthrough of the prototype. The co-created persona scenarios provided a narrative and insight into the lived experience of people with particular disabilities and health conditions, in a context and over time. This is in line with [14] who conducted similar types of persona-scenario workshops with patients and clinicians for a primary care intervention. They also found that this method provided rich descriptions and argue that the quality and quantity of ideas that were identified were significant and illustrate the added value and strength of this method.

This ties back to and answers to the critique from Treviranus [29] that personas created from background data lack the lived experience of the individual. Since the users co-create the personas with the experts, the participants' lived experiences are major components of the persona scenarios that we created. We found that creating persona scenarios with users gave high realism, and users were eager and willing to share information that would have been difficult to talk about in a more personal way. Co-creating personas with users with relevant, rich experience and / or functional declines can therefore be one of several methods to gather deep insights into the lived experience of potential users of the tool. This should provide the designers and developers with a better understanding of issues an individual with disabilities lives with every day.

As previously pointed out (section 4) people with disabilities lie outside what is considered the average or typical user. We contend that there is no meaningful way to create an average person with disabilities. In inclusive and universal design, it is important to take into account diverse users with diverse needs and to make the design work for as broad a population as possible. A strategy to do this is to involve so-called "edge-users", "outliers" or "extraordinary users" in design activities [32]. These people can easily identify pain points in the current situation. The idea is that creating a solution to satisfy the universal design needs of such a persona will likely satisfy the 
needs of less demanding users as well. Treviranus [29] notes that the best people to have at the design table are people that have difficulty with a current solution. They are not invested in keeping the current solution, and they will help stretch or expand your design further. Co-creation with these people has the potential to spur new and innovative solutions $[29,32]$. Therefore we asked the users in our persona scenario workshops to envision a persona that had been "a little unlucky", with perhaps multiple disabilities or challenges.

\section{Conclusion}

We have discussed the issue of collecting and representing the needs of marginalized user groups to support universal and inclusive design, and challenges related to gaining deep insight into their lived experience. A main critique of the persona method is the rather challenging task of condensing quantitative information into a few personas, and that this process can create increased distance to users rather than increased understanding.

We found that co-creating persona scenarios with users provided a type of information that we could not derive from focus groups, usability walkthrough or prototype or design workshops. The co-creation of persona scenarios gave us deeper insight into the lived experience of people with particular disabilities and health conditions, in a context and over time. While co-creating with users may increase the realism of the persona scenarios, it comes at the cost of statistical representativity. However, striving for this type of representativity may lead to personas that are not coherent and believable, and thus lose some of its potentially strongest properties, such as its ability to create deeper understanding, empathy and focus.

We suggest letting go of the idea that personas should reflect a quantitative segment, and rather use co-creation of persona scenario with users as a meaningful way to bring forth and discuss relevant and potentially sensitive information needs with users. We conclude that inclusive and participatory persona scenario creation can be a feasible and effective method to supplement other qualitative and quantitative methods to uncover user needs for universal and inclusive design purposes.

\section{Acknowledgement}

The work with this paper was supported by the Research Council of Norway through the CAPABLE project. We are grateful to the CAPABLE consortium, the municipalities and non-governmental organizations, which helped with recruiting participants to our workshops, and last but not least to the workshop participants for their valuable contributions. 


\section{References}

1. The origin of personas, https://www.cooper.com/journal/2008/05/the_origin_of_personas/, last accessed 2020/01/25.

2. Pruitt, J., Grudin, J.: Personas: practice and theory, http://dx.doi.org/10.1145/997078.997089, (2003). https://doi.org/10.1145/997078.997089.

3. Miaskiewicz, T., Kozar, K.A.: Personas and user-centered design: How can personas benefit product design processes? Design Studies. 32, 417-430 (2011).

4. Adlin, T., Pruitt, J.: The Essential Persona Lifecycle: Your Guide to Building and Using Personas. Elsevier Inc (2010).

5. Grudin, J.: Why Personas Work: The Psychological Evidence. In: The persona lifecycle. Morgan Kaufmann/Elsevier (2006)

6. Bjerknes, G., Bratteteig, T.: User participation and democracy. A discussion of scandinavian research on system development. Scandinavian Journal of Information Systems. 7, 73-98 (1995).

7. Grudin, J., Pruitt, J.: Personas, Participatory Design and Product Development: An Infrastructure for Engagement. In: Proceedings of Participation and Design Conference (PDC2002). pp. 144-161. , Sweden (2002).

8. Pruitt, J., Adlin, T.: The Persona Lifecycle: Keeping People in Mind Throughout Product Design (Interactive Technologies). Morgan Kaufmann (2006).

9. Cooper, A.: The Inmates Are Running the Asylum: Why High Tech Products Drive Us Crazy and How to Restore the Sanity. Macmillan Publishing Co., Inc., Indianapolis, Indiana, USA (1999).

10. LeRouge, C., Ma, J., Sneha, S., Tolle, K.: User profiles and personas in the design and development of consumer health technologies. Int. J. Med. Inform. (2011). https://doi.org/10.1016/j.ijmedinf.2011.03.006.

11. Gonzalez de Heredia, A., Goodman-Deane, J., Waller, S., Clarkson, P.J., Justel, D., Iriarte, I., Hernández, J.: Personas for policy-making and healthcare design. In: Proceedings of the DESIGN 2018 15th International Design Conference. pp. 2645-2656. Faculty of Mechanical Engineering and Naval Architecture, University of Zagreb, Croatia; The Design Society, Glasgow, UK (2018).

12. Vincent, C.J., Li, Y., Blandford, A.: Integration of human factors and ergonomics during medical device design and development: it's all about communication. Appl. Ergon. 45, 413-419 (2014).

13. Donald, M., Beanlands, H., Straus, S., Ronksley, P., Tam-Tham, H., Finlay, J., Smekal, M., Elliott, M.J., Farragher, J., Herrington, G., Harwood, L., Large, C.A., Large, C.L., Waldvogel, B., Delgado, M.L., Sparkes, D., Tong, A., Grill, A., Novak, M., James, M.T., Brimble, K.S., Samuel, S., Tu, K., Hemmelgarn, B.R.: Preferences for a self-management e-health tool for patients with chronic kidney disease: results of a patient-oriented consensus workshop. CMAJ Open. 7, E713-E720 (2019).

14. Valaitis, R., Longaphy, J., Ploeg, J., Agarwal, G., Oliver, D., Nair, K., Kastner, M., Avilla, E., Dolovich, L.: Health TAPESTRY: co-designing interprofessional primary care programs for older adults using the persona-scenario method. BMC Fam. Pract. 20, 122 (2019).

15. Duque, I., Dautenhahn, K., Koay, K.L., Willcock, L., Christianson, B.: A different approach of using Personas in human-robot interaction: Integrating Personas as computational models to modify robot companions' behaviour. In: 2013 IEEE RO-MAN. pp. 424-429 (2013). 
16. Schulz, T., Skeide Fuglerud, K.: Creating Personas with Disabilities. In: Miesenberger, K., Karshmer, A., Penaz, P., and Zagler, W. (eds.) Computers Helping People with Special Needs. pp. 145-152. Springer Berlin Heidelberg (2012).

17. Sulmon, N., Slegers, K., Van Isacker, K., Gemou, M., Bekiaris, E.: Using Personas to capture Assistive Technology Needs of People with Disabilities. In: Persons with Disabilities Conference (CSUN), Date: 2010/01/22-2010/01/27, Location: San Diego (2010).

18. AEGIS - Open Accessibility Everywhere - Personas, http://www.aegisproject.eu/index.php?option $=$ com_content $\&$ view $=$ article $\&$ id $=63 \&$ Itemid $=53$, last accessed 2020/01/25.

19. Wöckl, Bernhard Yildizoglu, Ulcay Buber, Isabella Diaz, Belinda Aparicio Kruijff, Ernst Tscheligi, Manfred: Basic senior personas: a representative design tool covering the spectrum of European older adults. Presented at the Proceedings of the 14th international ACM SIGACCESS conference on Computers and accessibility, Boulder, Colorado, USA (2012).

20. Kelle, S., Henka, A., Zimmermann, G.: A Persona-based Extension for Massive Open Online Courses in Accessible Design. Procedia Manufacturing. 3, 3663-3668 (2015).

21. Andriella, A., Torras, C., Alenyà, G.: Learning Robot Policies Using a High-Level Abstraction Persona-Behaviour Simulator. In: 2019 28th IEEE International Conference on Robot and Human Interactive Communication (RO-MAN). pp. 1-8 (2019).

22. Bødker, S., Christiansen, E., Nyvang, T., Zander, P.-O.: Personas, people and participation: challenges from the trenches of local government. Presented at the (2012).

23. Chang, Y.-N., Lim, Y.-K., Stolterman, E.: Personas: from theory to practices. In: Proceedings of the 5th Nordic conference on Human-computer interaction building bridges - NordiCHI '08. p. 439. ACM Press, New York, New York, USA (2008).

24. Matthews, T., Judge, T., Whittaker, S.: How do designers and user experience professionals actually perceive and use personas? In: Proceedings of the SIGCHI conference on human factors in computing systems. pp. 1219-1228 (2012).

25. Anvari, F., Richards, D., Hitchens, M., Babar, M.A., Tran, H.M.T., Busch, P.: An empirical investigation of the influence of persona with personality traits on conceptual design. J. Syst. Softw. 134, 324-339 (2017)

26. Bennett, C.L., Rosner, D.K.: The Promise of Empathy: Design, Disability, and Knowing the "Other." In: CHI 2019. https://doi.org/10.1145/3290605.3300528.

27. Stickdorn, M., Hormess, M., Lawrence, A., Schneider, J.: This Is Service Design Methods: A Companion to This Is Service Design Doing. O'Reilly Media, Incorporated (2018).

28. Vincent, C.J., Blandford, A.: The challenges of delivering validated personas for medical equipment design. Applied Ergonomics. 45, 1097-1105 (2014).

29. Treviranus, J.: The Three Dimensions of Inclusive Design, Part Two**, https://medium.com/@jutta.trevira/the-three-dimensions-of-inclusive-design-part-two7cacd12b79f1, last accessed 2019/01/31.

30. Fuglerud, K.S.: Inclusive design of ICT: The challenge of diversity. University of Oslo (2014). https://doi.org/10.13140/2.1.4471.5844.

31. Janson, A.L., Moen, A., Fuglerud, K.S.: Design of the CAPABLE prototype: preliminary results of citizen expectations. In: Granja, C. and Solvoll, T. (eds.) Proceedings of the 17th Scandinavian Conference on Health Informatics. p. 181. Linköping Electronic Conference Proceedings (2019).

32. Fuglerud, K.S., Sloan, D.: The Link between Inclusive Design and Innovation: Some Key Elements. In: Lecture Notes in Computer Science. pp. 41-50 (2013). 